\title{
La instrucción diferenciada en la evaluación formativa de la matemática
}

\section{The differentiated instruction in the formative assessment of mathematics}

\author{
Acosta, Tannia G \\ Universidad Agraria del Ecuador, Ecuador \\ Universidad de Guayaquil, Ecuador \\ Paccha, Mónica D \\ Yulan, Haydeé C \\ Universidad Agraria del Ecuador, Ecuador \\ Barre, Denisse M \\ Unidad Educativa Balmara, Ecuador
}

Autor para correspondencia: acosta@uagraria.edu.ec; mpaccha@uagraria.edu.ec; hylan@uagraria.edu.ec; dbchinita@gmail.com

Fecha de recepción: 20 de julio de 2018 - Fecha de aceptación: 30 de agosto de 2018

Resumen: Las diferencias individuales que existen en los estudiantes de determinado grupo de aprendizaje, en muchos casos dificultan la labor docente por cuanto cada estudiante tiene una manera diferente de aprender, dependiendo de sus conocimientos previos adquiridos. La presente investigación fue realizada con el objeto de conocer cómo influye la instrucción diferenciada en la evaluación formativa de los estudiantes. Ésta fue de carácter experimental con la ayuda de la investigación cuantitativa, tomando como base la investigación de campo. Asimismo, se aplicó un diseño experimental post-test de Polya basado en las fases de resolución de problemas, formando equipos y elaborando documentos didácticos, de acuerdo a recomendaciones pedagógicas. Los resultados mostraron un claro alcance de escalas superiores de aprendizaje con respecto a las obtenidas con evaluaciones tradicionales. Se concluye que los documentos pedagógicos encargados de la instrucción diferenciada se constituyen en elementos importantes para el éxito de este tipo de aprendizaje, por lo que se recomienda emplear un aprendizaje basado en problemas y una evaluación continua que permita detectar las falencias oportunamente.

Palabras Claves: matemáticas; instrucción diferenciada; evaluación formativa; educación superior Abstract: The individual differences that exist in the students of certain learning group, in many cases obstruct teaching labor, because each student has a different way of learning, depending on their previous knowledge. This research was carried out in order to know how it affects the differentiated instruction in the formative assessment of students. This was of experimental type with the help of quantitative research, on the basis of field research. In addition, a post-test of Polya experimental design was applied, based on the phases of troubleshooting, forming teams and developing educational documents, according to pedagogical recommendations. The results showed a clear scope of higher scales of learning with regard to those obtained with traditional assessments. It is concluded that the pedagogical documents responsible for differentiated instruction constitute important elements for the success of this type of learning, so it is recommended to use a problem-based learning and a continuous assessment that allow to timely detect the shortcomings.

Key Words: mathematics; differentiated instruction; formative assessment; higher education 


\section{Introducción}

El presente proyecto fue diseñado con el objetivo de mejorar el aprendizaje de aquellos estudiantes que no tienen el nivel académico que requiere el año escolar en el que se encuentran matriculados. Esto debido a la diversidad de estudiantes con los que se cuenta dentro de un salón de clases los mismos que a su vez tienen diferentes estilos de aprendizaje, haciendo más complicado el trabajo del docente que es lograr que todos aprendan. Es importante para que el proceso enseñanza aprendizaje se lleve a cabo con eficacia, se considere que no todos los estudiantes aprenden de igual manera y al mismo ritmo.

En este contexto, el hacer uso de la instrucción diferenciada juega un papel muy importante al momento de evaluar puesto que ayuda al docente a conocer mucho más a sus estudiantes y lo que son capaces de lograr si se les enseña desde sus propios conocimientos enriqueciéndolos a través de la formación de grupos de trabajo en los cuales todos puedan aportar con ideas de planteamiento y resolución de problemas.

Hacer uso de una enseñanza mediante la aplicación de instrucción diferenciada ayudará a los estudiantes a potencializar sus saberes, pues ese detalle que hace falta considerar al momento de enseñar hará una gran diferencia a futuro, pues aunque al inicio pueda resultar en mayor trabajo para los docentes, ya que requiere de hacer hojas de trabajo de acuerdo a la realidad de los estudiantes del grupo que se maneja, además de estar pendientes en cada momento del avance de los mismos a través de: lluvia de ideas, debates, listas de cotejo, entre otras formas de evaluar; luego se les facilitaría el trabajo en clases pues los estudiantes habrán nivelado sus conocimientos y el docente podrá seguir avanzando contenidos. En este marco, lo que se necesita estudiar es: ¿De qué manera influye la instrucción diferenciada en la evaluación formativa de la matemática?

\section{Enseñanza diferenciada}

Debido a las diferentes formas de aprender de cada individuo es imprescindible que se enseñe de manera diferenciada de acuerdo al nivel y forma de aprender de cada estudiante, "una misma talla no les sirve a todas las personas" (Gregory y Chapman, 2007, p.8). Por tal motivo, no se debe enseñar a todos aplicando una sola metodología puesto que no sería posible llegar a cada uno de los estudiantes, ya que como se ha mencionado anteriormente no todos aprenden de la misma forma, razón por la cual no se podría cumplir con los objetivos de aprendizaje trazados en el currículo académico. "La enseñanza diferenciada se basa en que una sola forma de enseñanza no abarca a todos los estilos de aprendizaje con los que el docente se enfrenta diariamente en el aula" (González, M. 2012, p.20); por tanto, es importante que el docente trabaje en función de los estilos de aprendizaje, siendo prioritario para ello el dominio total de este tema.

"Los enfoques pedagógicos en el curriculum en alfabetización mediática e informacional reconocen que los perfiles de aprendizaje de los estudiantes varían constantemente. Los estudiantes prefieren modos de interiorizar, procesar y comunicar información de carácter visual, táctil o quinesiológico. Los enfoques también reconocen que la diferenciación debe ocurrir en términos de las formas en las que los contenidos son presentados a los estudiantes, en el que los alumnos se involucran y en 
cómo son motivados para producir como un método de comprobación del aprendizaje”. (Wilson, 2012, p.15-24).

En el método de enseñanza-aprendizaje se debe aplicar nuevas formas de trabajo ya sea individual o en equipo que permita que los educandos interactúen entre sí desarrollando en ellos nuevas habilidades de razonamiento lógico y crítico que los encamine a la solución de diversos problemas matemáticos, esto logrará que dichos estudiantes vayan perfeccionando su manera de razonar y de ver las cosas, provocando en ellos una superación tanto social como intelectual, así lo indica Tomlinson (2001) "proveer diferentes caminos para adquirir contenido, para procesar la información y para generar productos" (p.3).

\section{Modelo de enseñanza diferenciada}

Refiriéndose a la educación diferenciada, Pascual Morán, (2014) dice: "se trata de una educación inclusiva y personalizada que, además ofrece cabida para toda vertiente pedagógica capaz de atender poblaciones y problemáticas emergentes de una manera diferencial" (p.231). En este modelo el docente debe buscar la forma de adaptar el contenido a cada estudiante, haciendo un análisis previo de las diversas formas de aprender, pero de una manera equitativa sin llegar a la discriminación.

Todo docente debe acoger a sus alumnos con el mismo amor y predisposición para enseñar, ya que no está en capacidad de escoger con que estudiantes trabajar. Además, el maestro debe estar plenamente comprometido y seguro de su vocación por la docencia.

No existe motivo para discriminar a ningún estudiante y debido a que actualmente se emplea una educación inclusiva todos los estudiantes deben participar de igual manera, logrando una conexión entre ellos que potencialice sus aptitudes, de acuerdo a las exigencias que se requiere en la educación actual.

Según Gonzáles (2012) “Diferenciar la enseñanza significa conocer el nivel de aprendizaje con el que cuenta un estudiante, sus necesidades e intereses. Es entender la forma de aprender de ese individuo y ofrecerle estrategias de enseñanza que satisfagan tales necesidades y permitir que el individuo demuestre todo su potencial" (p.19).

El profesor tiene la obligación de diferenciar a sus estudiantes por los conocimientos previos que tiene cada uno y por su forma de aprender aceptando cada una de sus diferencias. Así lo afirma Tomlinson (2008) "En un aula diversificada, el educador acepta incondicionalmente a sus alumnos tal y como son, y espera que den de sí lo máximo posible" (p.31). De esta manera el docente debe también procurar en lo posible que los estudiantes se planteen ir aumentando gradualmente el nivel de complejidad de los ejercicios a resolver.

\section{Enseñanza diferenciada en Matemática}

La enseñanza de matemática está pasando por un cambio muy importante, porque se exige que el maestro utilice diferentes recursos para lograr que el estudiante capte todo el 
conocimiento que se imparte clase a clase. Lo que resulta por demás necesario, para que lograr despertar el interés de los discentes y que se logre el proceso de enseñanza-aprendizaje.

La enseñanza de la matemática aplicando instrucción diferenciada es de extrema importancia ya que considera los diferentes ritmos de aprendizaje de los discentes, aplicando diferentes técnicas que permitan que los estudiantes vayan avanzando de manera progresiva en cuanto a los contenidos de la materia. Es más, actualmente con el avance de la tecnología es posible que el docente pueda presentar diferentes opciones de enseñanza que van desde el uso de un proyector para presentar un video relacionado al tema a tratar en la clase, hasta el enseñar el buen uso de la Internet para estudiar o representar de manera virtual a través de los diferentes programas que permiten la resolución de ejercicios o problemas que anteriormente no era posible visualizarlos de manera concreta.

En matemática "la Enseñanza Diferenciada ofrece varias formas de aprendizaje, no se asume un nivel separado para cada aprendiz" (Tomlinson, 2001, p.2). El docente que aplica enseñanza diferenciada debe seleccionar y hacer uso de distintas estrategias que le permitan mejorar el rendimiento académico de los estudiantes dependiendo del grupo al que esté enseñando, ya que una de las características de la enseñanza diferenciada es que es flexible, es decir debe ajustarse a las necesidades de los estudiantes con los que se está trabajando, es por ello esencial que el docente conozca en sus discentes las fortalezas con el fin de potencializarlas y las debilidades para superarlas.

\section{Importancia de evaluación formativa en matemática}

La evaluación formativa "es la que se realiza durante el desarrollo del proceso de enseñanza-aprendizaje para localizar las deficiencias cuando aún se está en posibilidad de remediarlas, esto es, introducir sobre la marcha rectificaciones a que hubiere lugar en el proyecto educativo y tomar las decisiones pertinentes, adecuadas para optimizar el proceso de logro del éxito por el alumno" (Rosales, 2014, p.3).

En matemática más que en cualquier otra asignatura es esencial palpar diariamente que los discentes comprendan la clase, ya que todo cuanto se enseñe en la asignatura en mención será utilizado para el desarrollo de la clase siguiente, pues la matemática requiere de ciertos proceso que se van llevando a cabo de manera secuencial; por ejemplo no se puede dividir si no se sabe multiplicar, o no se puede sumar fracciones heterogéneas si no se sabe determinar el mínimo común múltiplo para lo cual a su vez también debe saber multiplicar y dividir, etc. Es por ello que la matemática no permite que los estudiantes avancen sino tienen el dominio de un tema a utilizar en un nuevo contenido. "Continua o Continuada, es una característica de la evaluación formativa ya que ésta requiere la atención constante y sistemática a todo el proceso de aprendizaje. Como la inercia de los exámenes tiende a dominar, la evaluación continua se ha entendido, con más frecuencia de la deseada, como el control de resultados a través de la realización de exámenes o pruebas con cierta regularidad; por tanto, diremos que la evaluación formativa es continua, pero no que todas las prácticas de evaluación continua se pueden entender como evaluación formativa" (Fernández, 2017, p.5). 
Los estudiantes, además de tener dominio de los procedimientos matemáticos deben ser capaces de argumentar y razonar el porqué de una respuesta en un ejercicio o problema, pues el simple resultado del mismo sin un análisis, carece de todo sentido, y precisamente en ello radica la importancia no solo de dar solución a un problema sino también de realizar el análisis correspondiente que le permita al estudiante desarrollar su pensamiento crítico.

"La evaluación formativa, bien hecha, puede apoyar a los profesores en la construcción de ambientes matemáticos ricos en el aula" (Schoenfeld, 2015, p.193). Todo ello se puede lograr de manera paulatina pues como ya lo hemos mencionado anteriormente generalmente se cuenta con un grupo de estudiantes heterogéneo, por lo cual no todos avanzarán al mismo ritmo. Según Martinez (2013) "Los alumnos son actores clave de la evaluación formativa. Por su acceso inmediato y constante a sus propios pensamientos, acciones y trabajos, son la fuente fundamental de una buena retroalimentación..." (p.12). Es por ello que se los debe evaluar constantemente. Asimismo, el maestro debe aplicar diferentes técnicas y recursos con la intención de realizar una clase amena e interesante para el estudiante, manteniéndolo con el mismo interés de principio a fin.

\section{Desarrollo}

La investigación se desarrolló en la Unidad Educativa Réplica Simón Bolívar, ubicada en el complejo habitacional Ciudad Victoria, kilómetro 14,5 de la vía Daule Calle 27 al noroeste de la ciudad de Guayaquil. La población estuvo conformada por los octavos años de Educación General Básica sobre la cual se estableció cálculos matemáticos pertinentes por lo cual se aplicó el estudio correspondiente. El grupo investigativo trabajó sobre la población aplicando procesos de muestreo, obteniendo una porción de la muestra que se sometió a actividades matemáticas e investigativas, con el propósito de que el trabajo de investigación recabe datos fiables respecto de la misma. A la muestra calculada se le aplicó pre test y post test, con el fin de establecer si existe influencia de la instrucción diferenciada sobre la evaluación formativa.

Los datos se tabularon estadísticamente, para lo que se usó tablas y gráficos pertinentes. Después de este procedimiento se realizó un análisis de datos en interpretación de resultados a modo de contrastes de frecuencias usando chi cuadrado, con esto se determinó la influencia de la instrucción diferenciada sobre la evaluación formativa.

\section{Tipos de investigación}

La presente investigación corresponde a un trabajo experimental porque se trabajó con un reducido grupo de personas a las que se les realizó preguntas enfocadas directamente, partiendo de la formulación de hipótesis las cuales fueron aceptadas luego de la recolección y análisis de datos, y al elaborar las conclusiones.

Asimismo, se aplicó la investigación correlacional porque se ajustó al trabajo a realizar dado que lo que se buscaba obtener eran datos que sostengan si existe o no relación entre las variables: instrucción diferenciada y evaluación formativa. Lo que precisamente tiene como objeto la investigación correlacional, determinando si hay correlación positiva, negativa o si las variables no tienen correlación. 
Se necesitó también de la ayuda de la investigación cuantitativa ya que al estar realizando una investigación experimental es menester evaluar los resultados de forma numérica y concluyente al tabular los datos de la investigación en mención. La aplicación de la investigación cuantitativa facilitó la facultad de interpretar dichos resultados de una forma más sencilla llegando a una conclusión más asertiva.

Esta investigación tomó además como base la investigación de campo porque va de la mano con la investigación experimental y por ende con la cuantitativa, permitió mayor fiabilidad en los datos recolectados ya que dicho estudio se aplicó directamente sobre la población requerida a través del análisis de resultados obtenidos de la muestra de dicha población correspondiente a los octavos años de Educación General Básica de la Unidad Educativa Réplica Simón Bolívar.

\section{Población y muestra}

La población objeto del estudio, fue los estudiantes de octavo año de la Institución Educativa antes mencionada, en este caso el estudio experimental se aplicó directamente a la misma, debido a que, siendo una población pequeña, no es recomendable desde el punto de vista estadístico, calcular muestra de poblaciones de ese tipo. Los datos a continuación:

\begin{tabular}{lcc}
\multicolumn{3}{c}{ Tabla 1. Distribución de población } \\
\hline ESTRATO & POBLACIÓN & PORCENTAJE \\
\hline Estudiantes & 70 & $100 \%$ \\
\hline
\end{tabular}

\section{Diseño experimental post - test}

Debido a que los investigadores tuvieron acceso a las evaluaciones formativas de los bloques curriculares ya estudiados por los sujetos experimentales, se tuvo la necesidad únicamente de aplicar un diseño experimental pos test, de manera que se aplicó en los estudiantes la variable independiente, es decir, se formaron equipos acordes a las recomendaciones pedagógicas. Se elaboraron documentos didácticos para el trabajo en grupos con el fin de lograr un aprendizaje basado en problemas, es así que posteriormente se evaluaron los resultados obtenidos desde la perspectiva problemática, por lo que se diseñó un post test basado en las fases de resolución de problemas de Polya, en dónde; la primera fase consiste en llegar a la comprensión del problema; la segunda y tercera es la planificación/ejecución que consiste en construir un plan de resolución mediante representaciones simbólicas para después ser ejecutada; y la última fase es la verificación que es aquella que comprueba los resultados (Cobo \& Molina, 2014).

Como es sabido, los resultados evaluativos en la Educación General Básica tienen sus propias escalas de valoración, y fueron las mismas escalas de valoración las que se consideraron para expresar los resultados del pos-test, éstas se citan a continuación a la vez que en la tabla 2 , resume los resultados obtenidos; SAR: $\quad$ Supera los aprendizajes requeridos, DAR: Domina los aprendizajes requeridos, AAR: Alcanza los aprendizajes requeridos, PAAR: Está próximo a alcanzar los aprendizajes requeridos NAAR: No alcanza los aprendizajes requeridos. 
Tabla 2. Resultados diseño experimental pos-test.

\begin{tabular}{rrrrrrr}
\hline & SAR & DAR & AAR & PAAR & NAAR & TOTAL \\
\hline Sin instrucción diferenciada & 9 & 8 & 6 & 11 & 2 & 36 \\
Post test & & & & & & \\
Con instrucción diferenciada & 18 & 0 & 10 & 6 & 0 & 34 \\
TOTAL & 27 & 8 & 16 & 17 & 2 & 70 \\
\hline
\end{tabular}

El gráfico estadístico que resume los resultados y que facilita la observación de los mismos, a continuación:

Fig.1. Gráfico estadístico contraste de frecuencias obtenidas grupo experimental y de control.

\section{Contraste de variables}

El método estadístico utilizado para contrastar las variables y comprobar hipótesis fue Chi cuadrada definida por:

$$
x^{2}=\Sigma \frac{(f-f t)^{2}}{f t}
$$

Una vez aplicado a los datos obtenidos se logró:

$$
x^{2}=15,43
$$

Por otra parte, tenemos:

$$
\boldsymbol{x}^{2} \text { de la tabla }=\quad 9,4877
$$

Definimos nuestras hipótesis:

- Hipótesis nula, $H_{0}$ : La instrucción diferenciada no influye en la evaluación formativa de la matemática.

- Hipótesis alternativa, $H_{1}$ : La instrucción diferenciada si influye en la evaluación formativa de la matemática.

Siguiendo las reglas de este contraste de variables tenemos:

$$
\begin{gathered}
x^{2} \text { calculado }>x^{2} \text { de la tabla: se rechaza } H_{0} \\
x^{2} \text { calculado }<x^{2} \text { de la tabla se rechaza } H_{1}
\end{gathered}
$$

Como $\boldsymbol{x}^{2}=15,43>\boldsymbol{x}^{2}=9,4877$ : se rechaza $H_{0}$ y se acepta $H_{1}$, en tal virtud, La instrucción diferenciada si influye en la evaluación formativa de la matemática. 


\section{Conclusiones}

Luego del estudio aplicado, la prueba estadística para contraste de variables cualitativas Chi cuadrado, se demostró que efectivamente, la instrucción diferenciada logra una diferencia notable en la calidad del aprendizaje, pues gráficamente notamos que los resultados crecen dramáticamente en la escala máxima que es supera los aprendizajes requeridos, así también en la escala media de alcanza los aprendizajes requeridos.

Los documentos pedagógicos encargados de la instrucción diferenciada en sí, se constituyen como uno de los elementos más importantes para el éxito en este tipo aprendizaje, ya que lo que se intenta lograr con ellos es alcanzar un aprendizaje significativo lo más equiparado posible, es decir que las concepciones adquiridas sean empleadas en la resolución de problemas lo más cercanos a la realidad del estudiante, por lo que, éstos documentos debieran diseñarse y enfocarse en el aprendizaje basado en problemas.

Además, es imprescindible seguir evaluando a los estudiantes continuamente durante cada período de clase, con el objeto de identificar cualquier falencia que se presente respecto al aprendizaje de los estudiantes y de esta manera corregir aquello a tiempo. Para lo cual es importante también hacer uso de las guías didácticas que hemos desarrollado como propuesta del proyecto con el propósito de hacer más fácil tanto la tarea del maestro como la de los discentes. 


\section{Bibliografía}

Carmona Rodríguez, C., Sánchez Delgado, P., \& Bakieva, M. (2011). Actividades Extraescolares y Rendimiento Académico: Diferencias en Autoconcepto y Género. Revista de Investigación Educativa, 450.

Carreño González, I. (2008). Metodologías del aprendizaje. Madrid-España: Cultural S.A. Cevallos Veintimilla, M., \& Pilco Guailla, F. (2012). Las tareas extracurriculares para desarrollar la inteligencia lógico matemático. Bolívar, Ecuador: UNIVERSIDAD ESTATAL DE BOLÍVAR.

Chacón, M. (diciembre de 2014). Desarrollo de habilidades básicas para la construcción numérica, el cálculo y las relaciones matemáticas. Artículo, 32.

Cobo Lozano, P., \& Molina Hernández, A. (2014). ¿Pueden nuestros estudiantes construir conocimientos matemáticos? Revista de Didáctica de las Matemáticas, ISSN: 1887-1984, Vol.85

"Números", p. 50.

Fernández, S. (2017). Evaluación y aprendizaje. Revista de Didáctica ELE-ISSN 1885-2211. Núm.24, p.5

García Naranjo, C. (2007). Pedagogía moderna del aprendizaje. Guayaquil - Ecuador: Colección pedagógica.

Godino, J. D., Batanero, C., \& Font, V. (2003). Fundamentos de la enseñanza y el aprendizaje de la matemática para maestros. En J. D. Godino, Matemáticas y su didáctica para maestros (pág. 22). Granada: ReproDigital. Facultad de Ciencias.

González Cubero, M. (2012). El modelo de enseñanza diferenciada y su eficacia en los procesos de enseñanza y aprendizaje del idioma Español como lengua extranjera. Trabajo de graduación. Universidad Estatal a distancia. Maestría profesional en Psicopedagogía, San José, Cosa Rica, p. 19- 20

Gonzáles Arévalo, C. (2000). Un modelo de autogestión de las actividades extraescolares en un centro de educación secundaria. Educación física y deportes, p31.

Gregory, G. y Chapman, C. (2007). Differentiated Instructional Strategies. One size doesn’t fit all. Corwin Press.

López González, D., \& Rodrigo Hitos, J. (2011). Las competiciones de estudiantes como recurso didáctico en la enseñanza y aprendizaje de las matemáticas. Revista de formación e innovación educativa universitaria, 237.

Martínez Rizo, F. (2013). Dificultades para implementar la evaluación formativa. Perfiles educativos, vol.35, Nro.139, México 2013, p.12 
Pascual-Morán, A. (2014). Pedagogía de las diferencias y la equidad desde y hacia una educacióncultura inclusiva de paz positiva e integral. Vol. 10, Nro.2. Edición especial, 227-276. ISSN 1665-0441, p. 231

Rosales Mejía, M. (2014). Proceso evaluativo: evaluación sumativa, evaluación formativa y assesment, su impacto en la educación actual. ISBN-978-84-7666-210-6, Artículo 662. Ed. D, Caguas, Puerto Rico, p.3.

Tomlinson, C. A. (2000). Differentiation of instruction in the elementary grades. ERIC Digest. Champaign, IL: ERIC Clearinghouse on Elementary and Early Education.

Tomlinson, C. A. (1999). The Differentiated classroom. Responding to the needs of all learners. VA: ASCD

Wilson, C. (2012). Alfabetización mediática e informacional: proyecciones didácticas. Revista científica de educomunicación, Nro.39, V. XX2012. ISSN: 1134-3478, p. 15-24 